\title{
A culture for learning
}

\author{
M. Grace'
}

\section{Training and \\ development of the individuals in any organisation should be carried out for the benefit of both the organisation and the individuals, ultimately to achieve the objectives of that organisation}

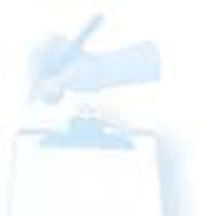

\section{In this part, we will} discuss:

$\checkmark$ Training

$\checkmark$ Education

$\checkmark$ Development
${ }^{1}$ Editor, British Dental Journal, 64 Wimpole Street, London W1G 8YS ${ }^{\star}$ Correspondence to: Mike Grace email:m.grace.bdj@bda-dentistry.org.uk REFEREED PAPER

(C) British Dental Journal

2001; 190: 469-472

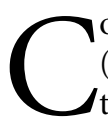
ontinuing Professional Development (CPD) has become the recognised way that professions can demonstrate willingness for their members to remain up to date in the skills and knowledge required to practise their profession ethically and responsibly.

Dentistry has seen a steady growth in the provision of appropriate educational initiatives to ensure that official undergraduate and postgraduate training and education are available.

Training and development of the individuals in any organisation should be carried out for the benefit of both the organisation and the individuals, ultimately to achieve the objectives of that organisation. We can be certain that one of these objectives will be the provision of quality service for the organisation's customers, which means that ultimately the customers (patients) will benefit. Thus training and development will help the individual, the organisation and the customers of that organisation. A good example of this is the Investors in People (IiP) recognition that is widely supported by governments in many countries.

This series looks at what constitutes best practice for organisations who have demonstrated effective training and development, and how the activities of these organisations can be adopted by everyone in dentistry. I believe adopting these activities will ensure greater benefit for organisations and individuals when participating in CPD than simply satisfying the mandatory requirements for recertification.

\section{Some definitions}

One of the difficulties with the whole area of education, training and development is the lack of clarity in the meaning of many of the words. The difference between education and training is often unclear, as it is between training and development.

One reason for this lack of clarity is the fact that the meanings themselves have changed over time, becoming more and more blurred as thinking in these areas has changed. In the past education tended to focus on the provision of knowledge in order to prepare people for their life in the world, with attempts to encourage thinking and study within people as well as to instigate questioning of beliefs and attitudes. By contrast training tended to refer to the transference of skills to enable people to carry out activities, either at work, in sport or in similar situations. Development was a much 'fuzzier' word and usually referred to senior management who were being developed (whatever that meant) for changes in the future in the organisation.

Buckley and Caple suggest the following definitions: ${ }^{1}$

- Training is a planned and systematic effort to modify or develop knowledge/skill/attitude through learning experience, to achieve effective performance in an activity or range of activities.

- Education is a process and series of activities which aim at enabling an individual to assimilate and develop knowledge, skills, values and understanding that are not simply related to a narrow field of activity but allow a broad range of problems to be defined, analysed and solved.

- Development is the general enhancement and growth of an individual's skills and abilities through conscious and unconscious learning.

The above definitions, while useful in themselves, become less important when people are actually planning how to learn more, either to solve a problem, improve performance or just achieve an objective. In the work environment (which is obviously the environment for CPD) most people probably require a blend of education, training and development in order to satisfy their needs for continuing learning to enable them to do what needs to be done.

The Institute of Personnel and Development (IPD) has produced a series of books called Training Essentials and the first in the series (Introduction to Training) suggests that the definitions of education, training and development are now so blurred that it is better to focus on learning for each individual and to ask 'What learning is required and when?' rather than 'What education, training or development should be provided?' This seems a sensible approach as it is much more 'learner-focused'.

\section{Continuing professional development}

No one can doubt that members of a profession have a duty and a responsibility to ensure their advice and care for the public is appropriate, relevant and the best available. It is the least people expect, and in the past has been traded for the freedom, status and other rewards that 
the professions have enjoyed. With time the expectations of the public have changed, and an increasing trend in consumer awareness has resulted in the need for the professions to be seen to be ensuring that their members are indeed maintaining their knowledge and skills to enable them to continue to provide appropriate levels of care.

\section{Lifelong learning}

In dentistry the GDC has adopted the term Lifelong Learning for the introduction of mandatory CPD. Lifelong learning requires all those on the Dentists Register to participate in 250 hours of CPD over a 5-year period, of which 75 hours must be verifiable and the remainder general. The verifiable hours simply means they can be verified by some means. The GDC recommends that these hours are divided equally within the 5 -year period, resulting in $\mathbf{5 0}$ hours a year.

No one can doubt that members of a profession have a duty and a responsibility to ensure their advice and care for the public is appropriate, relevant and the best available

\section{A more productive} belief is that teaching means creating a learning environment, and facilitating learning. The actual learning itself can obviously only be the responsibility of the learner.
The model most professions have adopted is the recertification model, whereby all those registered with the governing body (in the case of dentistry the General Dental Council, GDC) must satisfy the body that they are maintaining their knowledge and skills. The vehicle used for this maintenance of knowledge and skills is usually referred to as continuing professional development (CPD) or continuing professional education (CPE).

In dentistry the GDC has adopted the term Lifelong Learning for the introduction of mandatory CPD. Lifelong learning requires all those on the Dentists Register to participate in 250 hours of CPD over a 5-year period, of which 75 hours must be verifiable and the remainder general. The verifiable hours simply means they can be verified by some means. The GDC recommends that these hours are divided equally within the 5year period, resulting in 50 hours a year.

Guidance is provided in the literature on what will count as verifiable $\mathrm{CPD},{ }^{2}$ but the GDC has been careful to stress that the activities involved in CPD are up to the individual, and that all activities that result in learning will be considered to be appropriate.

Thus the stage is set for a new and organised approach to learning and development for all those involved in dentistry, but especially for dentists who appear on the Dentists Register.

\section{A culture of learning}

Although the guidance from the GDC focuses on learning as a means of satisfying the recertification requirements, the existing culture for providing opportunities and activities for postgraduate education still focuses more on teaching than learning. This is partly the result of the traditional approach to education that every- one has experienced and also the perceived requirements of those who wish to be taught (or to learn).

Before analyzing the relevance of these statements let us first consider the existing educational system in dentistry today as it is. Despite some very encouraging moves away from this traditional model, there is still a tendency for the postgraduate model for dental education and training to contain the following areas:

- An emphasis on teaching rather than learning.

- An emphasis on the providers of education rather than the recipients. Although this is slowly changing, most organised postgraduate activities still tend to focus on what course organisers can provide in the way of postgraduate lectures.

- A lack of coherence in planning of activities for all members of the dental team, and for dentists on the various groups themselves (community, general practice, hospital, etc.)

- A lack of focus on outcomes for the participants, including evaluation of the value of the activity or event.

- No attempt to match identified training needs with learning activities.

As already stated this state of affairs is partly historical, partly because it is always easier to continue doing what we have always done, and partly because of the perceived requirements of those being educated/trained/taught. Let us consider this last group first, in true customerfocused fashion.

\section{Responsibility of the learner}

Some people seem to believe that the teacher (for want of a better word) has responsibility for ensuring that the pupil learns. That belief is not only patently virtually impossible, but also counterproductive from the viewpoint of both learner and teacher. A more productive belief is that teaching means creating a learning environment, and facilitating learning. The actual learning itself can obviously only be the responsibility of the learner.

This basic truth seems to have become obscured in many learning activities, especially those involving the more traditional lecturing approach. The problem lies with the desire by all of us to learn as easily and conveniently as possible - especially in today's 'instant soundbite' world. We want to know, or we want to be able to do it - and we want it now.

This is the 'cook book' approach to learning so beloved by the instant expert, the guru and the 'How to do anything in a weekend and in five easy steps' course organiser. People are attracted to these courses that promise so much and deliver so little, apart from the fact they are almost always entertaining. They have to be - 


\section{continuing professional development}

because that is all they really offer. But does real learning take place?

As stated earlier we have to face up to the fact that while learning is fun, is sometimes easy and sometimes very hard, will invariably challenge us and is almost always highly stimulating - it is always our responsibility to seek it and work at it. Knowing our own learning style helps (see Part 7 in this series) but we have to be prepared to put effort in, and what we get out depends on how much we put in.

All of this comes back to the fact that people still feel that attending a lecture is easier and more acceptable than many other forms of learning, so lectures are still attended by many dentists in the hopes they will actually learn from them. In some cases some people do, but the potential for much greater learning is lost if that is all we do.

\section{Who decides?}

Another of the points described above is the fact that the decision as to what is available for people still tends to rest with the providers. People expect someone to put on a course or give them a book list to read. In response to this course organizers often canvas for opinions on what people want and then try to satisfy the perceived need by providing courses in areas people claim they want. The problem with this approach is that course organizers usually focus on process rather than outcome, in other words they do not enquire 'why' individuals state they want a course on a subject but prefer to simply ask 'what' do people want.

In other words providers often ask people if they want subject areas (rather than the actual outcome). For example questionnaires ask people to select topics (such as endodontics, orthodontics, practice management, etc.) because lecturers tend to be grouped by their chosen specialty. Few organisers ask what people would like to be able to do as a result of the learning activity (a daunting question) such as increase the number of patients accepting treatment plans, or provide a motivating environment for the development of staff.

Although the analogy is not completely accurate this process of asking people what subjects they would like rather than what outcomes they want is rather like dentists asking patients whether they would prefer a bridge, denture or implant rather than asking what outcome the patient wants (in other words to fill a gap, eat more effectively or prevent further problems). In the same way that it is up to the dentist's skill and experience to advise on the best way of achieving that outcome, surely it is up to the educator's or organiser's ability to choose how best to help dentists achieve the outcomes they want from learning.

\section{What do I want?}

Now we come to the real problem in existing learning activities, and paradoxically the reason why so much more benefit can be achieved.

Most dentists and dental teams actually do not know what they want. The traditional response to the question 'What are you hoping to get out of this course?' when asked at the start of almost any learning event (such as a lecture, seminar or workshop) is 'I'd like to learn something about the ... (insert the topic of the day)' or 'If I can pick up a few tips I'll feel the day was worth it.' The response to the first reply should be 'Why do you want to learn something about the ...(topic of the day)' but this is usually perceived as a threatening question and as a result is usually not asked as it can alienate the audience fairly rapidly. As for the comment '... pick up a few tips ...' the only possible response is that this is an incredible waste of time and money if that is all someone hopes to achieve for a full day of learning.

The concept of choosing objectives for a learning activity is fairly revolutionary for most people (not just dentists). This is partly because the concept of objectives is also fairly

\section{Steps to learning}

I. Learning is more effective if the provision of learning activities focuses more on learning than teaching, and on the recipients of learning rather than the providers.

2. Learning should focus on the outcome rather than the process, and the providers should have the knowledge and skill to provide appropriate learning methods depending on the learning styles of the recipients and the outcomes they want.

3. Learning will be more effective if the learners know what they want to learn, why they want to learn it, and what to do afterwards (in other words have some objectives for the learning).

unusual, despite the fact that the idea of setting out on a journey without having a clear idea of where you are going is fairly obvious. Thus any kind of learning with a clear objective must be more beneficial and valuable than simply turning up in the hope you will 'pick up a few tips.'

\section{A plan for training}

Planning is not always appreciated in business terms. Many people equate business plans with borrowing money, because banks require some form of 'plan' to satisfy head office before the loan can be considered. Many smaller businesses only ever plan when they are intending 


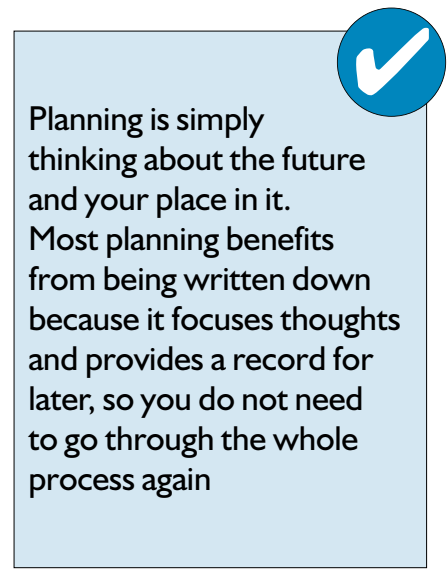

1 Buckley R, Caple J. The theory and practice of training. 4th ed. London: Kogan Page, 2000.

2 General Dental Council. Lifelong learning-taking dentistry forward. London: General Dental Council. borrowing money, or at least only ever write down a plan at such times.

Planning is simply thinking about the future and your place in it. Most planning benefits from being written down because it focuses thoughts and provides a record for later, so you do not need to go through the whole process again. Business planning is thinking about the role of the business in the future, trying to anticipate threats and take advantage of opportunities, and also predicting how the future may change and the relevance of any future changes to the business.

All of these activities are useful for any organisation, large or small. One aspect of planning that would seem essential is planning the effective use of resources, such as time and money. However planning the use of the most expensive resource for most organisations (the people in it) is often neglected.

In order to maximize on the effective use of people within an organisation (which usually also happens to be the best way of keeping those people motivated) you need a plan for training and developing them. This ensures they can carry out their jobs effectively and more easily, and also keeps them interested and motivated. So a training and development plan would seem an essential part of planning. Yet few organisations put their training plan in the same category as their marketing plan or their financial plans.

\section{Analysing the need}

The final piece in this jigsaw is the subject of the remainder of the articles in the series - the training needs analysis.

If we follow the argument up to now, we have stated the following steps:
- Learning is more effective if the provision of learning activities focuses more on learning than teaching, and on the recipients of learning rather than the providers.

- Learning should focus on the outcome rather than the process, and the providers should have the knowledge and skill to provide appropriate learning methods depending on the learning styles of the recipients and the outcomes they want.

- Learning will be more effective if the learners know what they want to learn, why they want to learn it, and what to do afterwards (in other words have some objectives for the learning).

The final step is simple. We must identify how much people already know and how competent they are at doing what needs to be done in order to do their jobs. If this knowledge is integrated with the priorities and objectives of the organisation then we will have an effective system for ensuring that learning is focused on the right people and in the right subjects.

This is known as a training needs analysis, and is outlined in the next article.

\section{Conclusion}

Whether we are considering training as a part of a programme of continuing professional development (CPD) or simply as a process for people in a business or organization (including obviously a dental practice) we should be focusing on the importance of ensuring that any learning has a clear reason (a training objective) which fits into the future plans of the business or organization. We should also ensure that the learning is as effective as possible and that something happens as a result which can be considered to be a favourable outcome. 